\title{
Robust Adaptive Beamforming Based on the Effectiveness of Reconstruction is
}

\author{
Yuping Zhang**, Yunjie Li*, Meiguo Gao* \\ Beijing Institute of Technology, Beijing,China
}

\begin{abstract}
The beamformers by joint estimation of the steering vector (SV) and reconstruct the interference-plus-noise covariance (INC) matrix have been investigated. However, few of them concerned about the effectiveness of reconstruction. In this paper, a novel beamformer is proposed, which introduces the improve factor (IF) to evaluate the improvement scale of output signalto-interference-plus-noise ratio (SINR) with and without reconstruction. By exploiting the relationship between the IF and input signal-to-noise ratio (SNR), it can be made a trade off between the extent of the performance improved and computational load that reconstruction required. Therefore, the SV is estimated at first, and then the level of SNR determines whether the INC matrix is required to reconstruct. During the implementation, a spherical uncertainty set and the sparsity of interference direction are used. Simulation results indicate that the proposed beamformer has better perfor-
\end{abstract}

\footnotetext{
This work is supported by the National Science Foundation of China (61406124), the Key Technology Research of space surveillance network sensor scheduling efficiency.

${ }^{*}$ Yunjie Li

** Yuping Zhang

Email addresses: sunshineacademic@163.com (Yuping Zhang), liyunjie@bit.edu.cn (Yunjie Li ), meiguo_g@bit.edu.cn (Meiguo Gao)
} 
mance compared with existing adaptive beamforming algorithms.

Keywords:

Covariance matrix reconstruction, improve factor, steering vector estimation

\section{Introduction}

Adaptive beamforming plays an important role in many fields of applications like radar, sonar, wireless communications, microphone array speech processing, medical imaging, and radio astronomy [1]. The well-known Capon adaptive beamformer has superior performance on interference-plus-noise suppression compared with the data-independent beamformers [2]. However, it suffers severe performance degradation due to mismatches between the presumed and actual steering vector (SV), the presence of signal of interest (SOI) in training data, and small training snapshots [3]. In order to address above problems, various robust adaptive beamformers have been develope$\mathrm{d}$ based on different principles. They can be categorized into the following categories: diagonal loading (DL) $[4,5]$, eigenspace-based projection (ECB) [6], SV estimation based on uncertainty set as well as the joint estimation of interference-plus-noise covariance (INC) matrix reconstruction and SV [3] [4] [7] [8].

Although the beamformer based on DL is simple and widely used, in practice, an optimal DL factor is difficult to determine. The beamformer based on ECB [6] is to estimate the signal-plus-interference subspace, and obtain the desired SV by projecting the presumed SV on the subspace. However, its performance is limited in the low signal-to-noise ratio (SNR). Besides, it is sensitive to SV mismatches and imprecise knowledge of the INC matrix. The 
beamformer based on the spherical or ellipsoidal uncertainty set surrounding the nominal SV [3] [4] [7] [8], can be robust against the SV mismatch by solving an optimization problem. Whilst, as the input SNR increases, the presence of SOI in sample covariance matrix leads to the significant performance degradation. In order to improve the robustness against above problems, a new type of beamformer based on combination of INC matrix reconstruction and $\mathrm{SV}$ is proposed $[9,10,11,12]$. In [9], the INC matrix reconstruction is performed based on the Capon spectrum by integrating over an angular sector where the interference SVs locate. However, in [10], by exploiting the sparsity of the directions of the source signals, the INC matrix was reconstructed by solving a compressive sensing (CS) problem. Although the performance of above beamformers in $[9,10]$ can be effective against signal direction error, the precise array manifold is required to provide. Practically, the array calibration errors always exist due to antenna location error, mutual coupling, and the channel error. In this case, the reconstruction operation only considers the pointing errors to ignore other potential errors. In [11], the high-dimensional annulus uncertainty set of interference SV was used to reconstruct the INC martix. Moreover, the SV is estimated by solving a quadratically constrained quadratic programming (QCQP) problem. In [12], the reconstruction operation takes array manifold error into account, however, it leads to a large amount of calculation without considering the sparsity of interference signal. More important, these beamformers based on reconstruction paid little attention to the effectiveness of reconstruction in different input SNRs.

In this paper, the idea of improve factor (IF) is introduced to evaluate 
the effectiveness of reconstruction. From the point of view of IF, a novel beamformer is proposed to improve robustness against SV mismatch and the covariance matrix contaminated by desired signal component. The major work is presented as the following three aspects. The first aspect is to introduce IF. By exploiting the relationship between the IF and the input SNR, we found that IF is small in low SNR, and in contrast, a large IF can be obtained in high SNR. The second one is to estimate the desired SV in advance, then the SNR is estimated. Because the SV has been estimated, the relatively precise signal power can be obtained. Instead of using convex optimization toolbox, the desired SV is estimated based on the spherical uncertainty. The noise power can be approximately calculated by the minimum eigenvalue of the sample covariance matrix. So far, the SV and SNR estimation are completed. The third one is to reconstruct the INC matrix based on the Capon spatial spectrum integration by making use of the sparsity of interferences direction. Meanwhile, the array calibration errors are taken into account.

The rest of this paper is organized as follows. The system model and some necessary background are demonstrated in Section 2. In Section 3, a new beamformer based on effectiveness of reconstruction is proposed. Then, the desired SV is estimated based a spherical uncertainty. Next, the INC matrix is reconstructed by making use of sparsity of interferences distribution. In Section 4, simulation results are presented. Finally, some conclusions are drawn in Section 5. 


\section{System model and Problem background}

By considering a uniform linear array consisting of $M$ isotropic antenna elements spaced by half wavelength, the instantaneous output of a narrowband beamformer at time $k$ is expressed by [3]

$$
y(k)=\boldsymbol{w}^{H} \boldsymbol{x}(k)
$$

where $\boldsymbol{w} \in \mathcal{C}^{M \times 1}$ denotes the complex weight vector. The symbol $(\cdot)^{T}$ and $(\cdot)^{H}$ denote the transpose and Hermitian transpose, respectively. The array observation vector $\boldsymbol{x}(k)$ can be denoted as [3]

$$
\begin{aligned}
\boldsymbol{x}(k) & =s(k) \boldsymbol{a}+\boldsymbol{i}(k)+\boldsymbol{n}(k) \\
& =\boldsymbol{s}(k)+\boldsymbol{i}(k)+\boldsymbol{n}(k)
\end{aligned}
$$

where $\boldsymbol{s}(k), \boldsymbol{i}(k)$, and $\boldsymbol{n}(k)$ denote the statistically independent components of the desired signal, interference, and noise, respectively. The $\boldsymbol{a}$ is the SV corresponding to the direction of desired signal, $s(k)$ is the complex signal waveform. The optimal weight vector $\boldsymbol{w}_{\text {opt }}$ can be derived by maximizing the output signal-to-interference-plus-noise ratio (SINR) [8]

$$
\mathrm{SINR}=\frac{\sigma_{s}^{2}\left|\boldsymbol{w}^{H} \boldsymbol{a}\right|^{2}}{\boldsymbol{w}^{H} \boldsymbol{R}_{i+n} \boldsymbol{w}}
$$

where $\sigma_{s}^{2}$ represents the desired signal power, and and $\boldsymbol{R}_{i+n} \in \mathcal{C}^{M \times M}$ is the INC matrix. As can be derived that, the maximization of (3) is equivalent to the Capon beamformer

$$
\min _{\boldsymbol{w}} \boldsymbol{w}^{H} \boldsymbol{R}_{i+n} \boldsymbol{w} \quad \text { s.t. } \quad \boldsymbol{w}^{H} \boldsymbol{a}=1
$$


From (4), the well-known solution can be expressed as follows

$$
\boldsymbol{w}_{\text {opt }}=\frac{\boldsymbol{R}_{i+n}^{-1} \boldsymbol{a}}{\boldsymbol{a}^{H} \boldsymbol{R}_{i+n}^{-1} \boldsymbol{a}}
$$

Correspondingly, the optimal SINR is given by [8]

$$
S I N R_{o p t}=\sigma_{s}^{2} \boldsymbol{a}^{H} \boldsymbol{R}_{i+n}^{-1} \boldsymbol{a}
$$

From (6), the output SINR is not only related to the desired signal steering vector $\boldsymbol{a}$, but also the INC matrix $\boldsymbol{R}_{i+n}$. Unfortunately, neither of them is easy to obtain in practice. The former is usually replaced by the nominal (presumed) one $\overline{\boldsymbol{a}}$, whereas, in practical circumstances, some mismatches between the nominal and actual SV can easily occur due to array imperfections such as look direction, signal pointing errors, array calibration errors, etc. If the INC matrix $\boldsymbol{R}_{i+n}$ is available, adaptive beamformer can be performed well against these mismatches. However, the $\boldsymbol{R}_{i+n}$ requires an infinite number of pure snapshots data without the desired signal. It is usually replaced by sample covariance matrix $\hat{\boldsymbol{R}}_{x}=\frac{1}{K} \sum_{k=1}^{K} \boldsymbol{x}(k) \boldsymbol{x}^{H}(k)$, where the $K$ is the number of snapshots. In this case, it may result in significant performance deterioration, especially in the high input SNR [6]. As stated above, it is necessary to estimate the desired signal SV and make the component of the SOI free from the sample covariance matrix, simultaneously.

From the standpoint of covariance matrix, recently, a robust adaptive beamformer (RAB) technique based on the following Capon spectrum over some special directions[13] 


$$
\hat{\sigma}^{2}(\boldsymbol{a}(\theta))=\frac{1}{\boldsymbol{a}^{H}(\theta) \hat{\boldsymbol{R}}_{x}^{-1} \boldsymbol{a}(\theta)}
$$

Assuming to have the precise array manifold, where the $\boldsymbol{a}$ is the actual SV with respect to the direction $\theta$.

However, it is hard to obtain precise prior information about the array manifold. The actual SV $\boldsymbol{a}(\theta)$ is usually replaced by the nominal SV $\overline{\boldsymbol{a}}(\theta)$. Based on the Capon spectrum estimator (7), the INC matrix $\tilde{\boldsymbol{R}}_{i+n}$ can be reconstructed as follows

$$
\tilde{\boldsymbol{R}}_{i+n}=\int_{\bar{\Theta}} \frac{\overline{\boldsymbol{a}}(\theta) \overline{\boldsymbol{a}}^{H}(\theta)}{\overline{\boldsymbol{a}}^{H}(\theta) \hat{\boldsymbol{R}}_{x}^{-1} \overline{\boldsymbol{a}}(\theta)} d \theta
$$

where $\bar{\Theta}$ is the interference and noise region selected as the complement sector of the desired signal region $\Theta$. From (8), the mismatch between the actual SV $\boldsymbol{a}(\theta)$ and the nominal one $\overline{\boldsymbol{a}}(\theta)$ may result in the imprecise estimation of $\boldsymbol{R}_{i+n}$. It notes that the there is no limit on reconstruction. If the input SNR is low, the advantage obtained from reconstructed matrix $\tilde{\boldsymbol{R}}_{i+n}$ is not obvious with respect to the sample covariance matrix. Hence, the effectiveness of reconstruction should be pay much attention. In (8), the nominal $\overline{\boldsymbol{a}}(\theta)$ should be replaced by actual one considering the array errors. Moreover, it is unnecessary that the whole region $\bar{\Theta}$ is used, because the interference distribution is sparse, most components in $\bar{\Theta}$ are noise.

\section{New approach to robust beamforming}

In this section, a new RAB beamformer based on effectiveness of reconstruction [4] is proposed. At first, the concept of IF is illustrated, the relationship between the IF and input SNR is exploited. Then, the actual 
SV associated with desired signal is estimated in the condition of the prior knowledge of desired signal region and imprecise array structure. Using the obtained desired signal SV, the relatively accurate signal power can be obtained by spectrum estimation, and the noise power is also approximately estimated by eigenvalues decomposition of the sample covariance matrix. So, the input SNR can be computed. Next, according to the estimated SNR, the INC matrix can be determined whether to reconstruct. Once the INC matrix is reconstructed, the sparsity of interference distribution can be used. Only the main interference SVs corresponding to the interference region will be corrected, and then participate in the integration based on the Capon spatial spectrum.

\subsection{The description of IF}

In this section, in order to evaluate the effectiveness of reconstruction, the concept of IF is proposed, and can be defined as follows

$$
\mathrm{IF}=S I N R_{\text {after }} / S I N R_{\text {before }}
$$

where the $S I N R_{a f t e r}$ and $S I N R_{\text {before }}$ are the with and without performing the INC matrix reconstruction, respectively. From the equation (6), the SINR is determined by two components, the one is the sample covariance matrix $\hat{\boldsymbol{R}}_{x}$, and the other is the desired SV $\boldsymbol{a}$. In order to facilitate the illustration, we assume that the desired SV $\boldsymbol{a}$ can be estimated by various algorithms [14] [7] [15]. Here, to facilitate the real-time processing and avoid using convex optimization toolbox, the algorithm based a spherical uncertainty set is introduced, and it will be detailed in Section 3.2. Hence, so far, only the INC matrix reconstruction will have impact on the output SINR. Therefore, 
the scale of improvement of reconstruction can be measured by IF. The larger IF is, the better effectiveness can be obtained by reconstruction. On the contrary, although the reconstruction is implemented, a little improvement of output SINR is obtained. From a number of experiments about adaptive beamformers, it can be found that IF is closely related with the input SNR. For example, the IF obtained by the output SNR of [12] to [4] is shown in Fig.1. It can be seen that the IF is relatively small in low SNR situations, whereas, in high SNR situations, the large value of IF can be achieved. It is understandable that since the sample covariance matrix mainly consists of interference and noise in the low SNR, it has the robustness against the SV mismatches. However, when the SNR is high, it means that the desired signal can be not ignored in the sample covariance matrix, therefore, the INC matrix reconstruction is required to remove it. Up to now, we need to know how to divide the bound of the low SNR or the high SNR as well as how to estimate the SNR.

It is well known that the low/high SNR is relatively concept. Thus, it is difficult to divide the bound of low/high SNR. As stated above, the division of SNR is closely related with IF. In this paper, considering a uniform linear array with 10 omnidirectional sensors and half-wavelength inter-element spacing [7][16][17], when the IF is less than $3 \mathrm{~dB}$, we regard that the reconstruction has a little effectiveness for improving the performance of beamformer. Thus, the corresponding SNR is $0 \mathrm{~dB}$. As can be asserted that IF helps to make a trade off between the extent of the performance improved and computational load that reconstruction required. For instance, assuming the constant computational amount, the current SNR is estimated -10dB, 




Figure 1: The relationship between the IF and input SNR

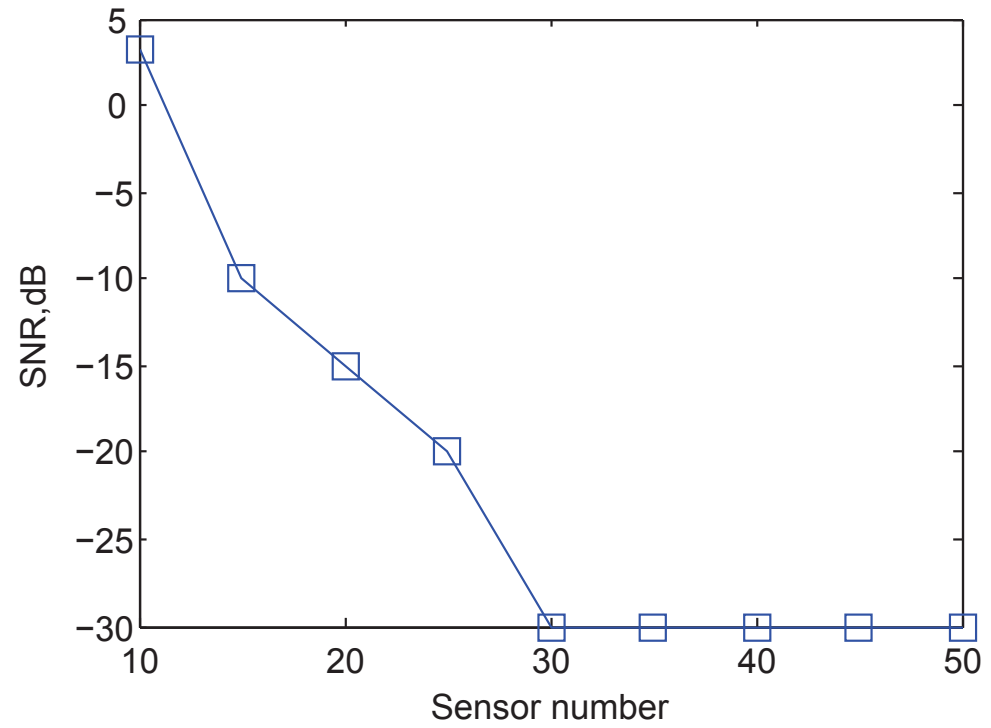

Figure 2: The bound of the low SNR versus sensor number 
even the reconstruction is performed, the performance improvement is less than $3 \mathrm{~dB}$. In contrast, if the $\mathrm{SNR}$ is $10 \mathrm{~dB}$, the performance improvement can be obtained about $10 \mathrm{~dB}$ with the same computational amount. Therefore, the bound of the low SNR is regarded as 0dB.

The bound of the low SNR is investigated as a function of the sensor number, and the results are shown in Fig.2. Note that the curve can be obtained by setting the IF as $3 \mathrm{~dB}$. It can be seen that the bound decreases as the sensor number increases, when the sensor number is less than 30 . Nevertheless, the bound almost remains about -30dB when the sensor number is larger than 30. That is to say, in case of 20 sensors, the input SNR is larger than $-15 \mathrm{~dB}$, the reconstruction operation is relatively effective in case of 20 sensors. Compared with 10 sensors, the corresponding bound of $\mathrm{SNR}$ is reduced about $18 \mathrm{~dB}$. Meanwhile, it indicates that the tolerance of the covariance matrix for containing desired signal is reduced as the sensor number increases, and the minimum input SNR is about -30dB when sensor number is larger than 30. In order to facilitate comparison, the sensor number is set as 10 as shown in subsequent experiments. Furthermore, the SNR estimation will be presented in the following section.

\section{2. $S V$ and SNR Estimation}

Using the similar idea of the beamformer in [4], the nominal SV can be corrected by solving the following

$$
\min _{\boldsymbol{a}(\theta)} \boldsymbol{a}^{H}(\theta) \hat{\boldsymbol{R}}_{x}^{-1} \boldsymbol{a}(\theta) \quad \text { s.t. } \quad\|\boldsymbol{a}(\theta)-\overline{\boldsymbol{a}}(\theta)\|^{2} \leq \varepsilon
$$

It is assumed that the actual SV $\boldsymbol{a}(\theta)$ is located in a sphere uncertainty set centered with the nominal SV $\overline{\boldsymbol{a}}(\theta)$, and the $\theta \in \Theta$, where $\varepsilon$ is the uncertainty 
level represented as Euclidean distance between the SV $\boldsymbol{a}(\theta)$ and $\overline{\boldsymbol{a}}$, and $\|\cdot\|$ denotes a vector norm. By applying the Lagrange multiplier methodology, we obtain

$$
L=\boldsymbol{a}^{H}(\theta) \hat{\boldsymbol{R}}_{x}^{-1} \boldsymbol{a}(\theta)+\lambda\left(\|\boldsymbol{a}(\theta)-\overline{\boldsymbol{a}}(\theta)\|^{2}-\varepsilon\right)
$$

where the $\lambda$ denotes the Lagrange multiplier. Computing the differentiation of (11) with respect to $\boldsymbol{a}(\theta)$ the desired SV can be obtained as follows

$$
\boldsymbol{a}_{0}(\theta)=\overline{\boldsymbol{a}}(\theta)-\left(\boldsymbol{I}+\lambda \hat{\boldsymbol{R}}_{x}\right)^{-1} \overline{\boldsymbol{a}}(\theta)
$$

where $\boldsymbol{I}$ is the identity matrix, and $\lambda$ can be solved from the equation $\|(\boldsymbol{I}+$ $\left.\lambda \hat{\boldsymbol{R}}_{x}\right)^{-1} \overline{\boldsymbol{a}}(\theta) \|^{2}=\varepsilon$. Once the $\lambda$ is obtained, according to equation (12), the actual desired SV $\boldsymbol{a}_{0}(\theta)$ is determined, and replacing $\boldsymbol{a}(\theta)$ with $\boldsymbol{a}_{0}(\theta)$ in (7), the desired signal power $\hat{\sigma}_{0}^{2}$ can be estimated. In order to eliminate the ambiguity in the SOI covariance term, using $\left\|\boldsymbol{a}_{0}^{2}\right\|=M$, therefore, the desired signal power can be further estimated as

$$
\hat{\hat{\sigma}}_{0}^{2}=\hat{\sigma}_{0}^{2}\left\|\hat{\sigma}_{0}\right\|^{2} / M
$$

It has been confirmed in [18] that the $\hat{\hat{\sigma}}_{0}^{2}$ is more accurate than $\hat{\sigma}_{0}^{2}$. Besides, the minimum eigenvalue of $\hat{\boldsymbol{R}}_{x}$ can be exploited to estimate the noise power $\hat{\sigma}_{n}^{2}$ as [19]. Hence, the input SNR can be defined as

$$
\widehat{S N R}=\hat{\hat{\sigma}}_{0}^{2} / \hat{\sigma}_{n}^{2}
$$

The numerical examples in [3] [20] have been shown that the output SINR of the most beamformers approximate optimum beamformer in (6) at the relatively low SNR, even though the sample covariance matrix $\hat{\boldsymbol{R}}_{x}$ is adopted. It stems from the fact that the output SINR reduces mainly due to the 
imprecise SV $\overline{\boldsymbol{a}}(\theta)$ in the low SNR condition, and the effect of $\hat{\boldsymbol{R}}_{x}$ with desired signal component can be neglected. In other words, only to estimate the actual SV, the performance can be improved obviously. Conversely, for the high SNR conditions, the performance improvement is limited by the virtue of SV estimation only. Therefore, the INC matrix reconstruction should be performed as described in the following section.

\subsection{Interference-Plus-Noise Covariance Matrix Reconstruction}

As stated above in Section 2, the core idea of INC matrix reconstruction is to accumulate the spatial power spectrum of interference-plus-noise over the angular sector contained interference and noise. In $[12,13]$, the $\bar{\Theta}$ includes all angular sector except the desired signal sector, which means arbitrary direction $\theta(\theta \in \bar{\Theta})$ as well as corresponding SVs will take part in the integral calculation. In practice, the large amplitude of spatial power spectrum is sparsely distributed surrounding the actual SV. Nevertheless, in other direction, the amplitude of spatial power spectrum is quite small. Using the whole sector $\bar{\Theta}$ may bring the redundancy integral information and extra computational costs. Instead, in this paper, applying the sparsity of interference sources in [10] and interference signal power estimation as aforementioned, the new INC matrix $\tilde{\boldsymbol{R}}_{i+n}^{\prime}$ can be reconstructed as

$$
\tilde{\boldsymbol{R}}_{i+n}^{\prime}=\tilde{\boldsymbol{R}}_{I}+\tilde{\boldsymbol{R}}_{N}
$$

where $\tilde{\boldsymbol{R}}_{I}$ and $\tilde{\boldsymbol{R}}_{N}$ are defined as

$$
\begin{aligned}
\tilde{\boldsymbol{R}}_{I} & =\int_{\theta \in \Theta_{I}} \hat{\hat{\sigma}}^{2}(\hat{\boldsymbol{a}}(\theta)) \hat{\boldsymbol{a}}(\theta) \hat{\boldsymbol{a}}^{H}(\theta) \quad d \theta \\
\tilde{\boldsymbol{R}}_{N} & =\int_{\theta \in \Theta_{N}} \hat{\sigma}^{2}(\overline{\boldsymbol{a}}(\theta)) \overline{\boldsymbol{a}}(\theta) \overline{\boldsymbol{a}}^{H}(\theta) \quad d \theta
\end{aligned}
$$


where the $\Theta_{I}$ and $\Theta_{N}$ are the interference and noise region, respectively. The $\hat{\sigma}^{2}(\hat{\boldsymbol{a}}(\theta))$ and $\hat{\sigma}^{2}(\overline{\boldsymbol{a}}(\theta))$ can be obtained by the (13) and (7), respectively. It notes that only the actual interference SVs are corrected before the SVs are integrated. Whilst, the SVs with respect to the noise region is unnecessary to correct due to noise nature. It is noted that the second term in (15) should be not identified as the whole noise covariance, because the interference components may contain some noise components.

Assuming the number of interference angular sector $P$, the $\Theta_{I}=\left\{\Theta_{I_{1}} \cup\right.$ $\left.\Theta_{I_{2}} \cup \cdots \Theta_{I_{P}}\right\}$ denotes the angular sector set of the interferences, which can be divided into small, discrete interferences sector $\Theta_{I_{p}}$. Where the $\Theta_{I_{p}}$ can be estimated by a spectral search algorithm [21] or a search-free method [22], here, the well known Capon spatial spectrum (7) is used for the direction of arrival (DOA) estimation. The actual interference SVs should be estimated as (12). That is to say the $\hat{\boldsymbol{a}}\left(\theta_{p}\right)$ should satisfy as follows

$$
\left\|\boldsymbol{a}\left(\theta_{p}\right)-\overline{\boldsymbol{a}}\left(\theta_{p}\right)\right\|^{2} \leq \varepsilon, \quad \theta \in \theta_{I}
$$

In order to cover each interference SV in the same uncertainty set, the uncertainty level factor $\varepsilon$ should satisfy

$$
\varepsilon \geq \max _{i=1,2, \ldots, P} \min _{\theta \in \Theta_{I}}\left\|\overline{\boldsymbol{a}}(\theta)-\boldsymbol{a}\left(\theta_{p}\right)\right\|^{2}
$$

Then, each interference SV can be corrected by

$$
\hat{\boldsymbol{a}}\left(\theta_{p}\right)=\overline{\boldsymbol{a}}\left(\theta_{p}\right)-\left(\boldsymbol{I}+\lambda \hat{\boldsymbol{R}}_{x}\right)^{-1} \overline{\boldsymbol{a}}\left(\theta_{p}\right) \quad \theta_{p} \in \Theta_{I_{p}}
$$

where $\lambda$ is a parameter related to $\varepsilon$, and can be solved according to (11). Finally, the INC matrix $\tilde{\boldsymbol{R}}_{i+n}^{\prime}$ can be obtained by

$$
\tilde{\boldsymbol{R}}_{i+n}^{\prime}=\sum_{p=1}^{P} \tilde{\boldsymbol{R}}_{I_{p}}+\tilde{\boldsymbol{R}}_{N}
$$


To sum up, the proposed method based on spherical uncertainty set is summarised as follows

Step 1. Estimate the desired signal SV $\hat{\boldsymbol{a}}_{0}(\theta), \quad \theta \in \Theta$ with (12) with a uncertainty set $\varepsilon_{0}$, and then compute input $\widehat{S N R}$ with $(7)(13)$ and (14), respectively.

Step 2. If the $\widehat{S N R} \leq 0$ is satisfied, using the $\hat{\boldsymbol{a}}_{0}(\theta)$ obtained in step 1 , and the sample covariance matrix $\hat{\boldsymbol{R}}_{x}$, calculate the adaptive beamformer weight $\boldsymbol{w}_{0}=\hat{\boldsymbol{R}}_{x}^{-1} \hat{\boldsymbol{a}}_{0} / \hat{\boldsymbol{a}}_{0}^{H} \hat{\boldsymbol{R}}_{x}^{-1} \hat{\boldsymbol{a}}_{0}$. Otherwise, perform the next step.

Step 3. Estimate the DOA according to (7), and divide the interference angular sectors $\Theta_{I}$ into some small ones $\Theta_{I_{p}}, \quad i=1,2, \ldots, P$. For each $\Theta_{I_{p}}$, the interference SV is firstly corrected with (19), and then to calculate $\tilde{\boldsymbol{R}}_{I_{p}}$ and $\tilde{\boldsymbol{R}}_{N}$, next, the INC matrix $\hat{\boldsymbol{R}}_{i+n}^{\prime}$ can be obtained with (20). Finally, calculate the adaptive beamformer weight $\boldsymbol{w}_{0}=\tilde{\boldsymbol{R}}_{i+n}^{\prime}{ }^{\prime} \hat{\boldsymbol{a}}_{0} / \hat{\boldsymbol{a}}_{0} \tilde{\boldsymbol{R}}_{i+n}^{\prime \prime} \hat{\boldsymbol{a}}_{0}^{H}$

Note that the major differences between the proposed method and the beamformer in [4][13][10], are presented in terms of the types of mismatches and computational complexity. The beamformer in [4] is emphasized to improve the robustness against uncertain steering vectors. In addition, this approach requires $O\left(M^{3}\right)$ flops. To make robust against the presence of desired signal in the training snapshots, the approach in [13] attempts to reconstruct the INC matrix. Since it does not impose a norm constraint on the steering vector, it has better robustness against the gain perturbations than the former as mentioned above. Its computational complexity is typically $O\left(M^{2} S\right)$ flops, assuming $S \gg M$, where $S$ denotes the number of sampling points in the area eliminating desired signal [13]. As can be seen, more robust performance should be paid for relatively high computational load. In [10], 
by making use of sparsity of the signal sources and the priori information of the directions of the source signals, the INC matrix is reconstructed based compressive sensing (CS) problem. As can be seen from the simulation results, it has robustness against the mismatch SV and uncertainty covariance matrix over a wide range of SNR. Moreover, its computational complexity is $O\left(N M^{2}\right)$ with $N \gg M$, where $N$ denotes the number of interested sample grids. From [10], one can find that the mainly computational costs on the spectral search. Compared with [13], the approach in [10] has low computational complexity, since it is commonly $S \gg N$. Similar to the approach in [10] in terms of the type of mismatches over a wide range of SNR. However, our approach is conditionally reconstruction. We highlight the effectiveness of reconstruction by IF. It can be seen that we make a fusion between the approach [4] and [13]. Meanwhile, the effectiveness of reconstruction is highlighted. Without reconstruction, the proposed method has approximately the computational complexity with that of [4]. By contrast, the complexity is approximately that of [13].

\section{Simulation results}

In this section, considering a uniform linear array (ULA), which consists of 10 isotropic sensors spaced with half-wavelength, there are two interferences with direction of $\theta_{1}=30^{\circ}$ and $\theta_{2}=70^{\circ}$, the interference-to-noise ratios (INRs) of them are set to $30 \mathrm{~dB}$. The additive noise is assumed to be spatially white Gaussian process with covariance matrix $\hat{\sigma}_{n}^{2} \boldsymbol{I}\left(\hat{\sigma}_{n}^{2}=1\right)$. The actual direction of desired signal is $\theta_{0}=0^{\circ}$. The angular sector of the interference signals and the SOI are assumed to be $\Theta_{I}=\left[\bar{\theta}_{1}-5^{\circ}, \bar{\theta}_{1}+5^{\circ}\right] \cup\left[\bar{\theta}_{2}-5^{\circ}, \bar{\theta}_{2}+5^{\circ}\right]$ 
and $\Theta=\left[\bar{\theta}_{0}-5^{\circ}, \bar{\theta}_{0}+5^{\circ}\right]$. For each scenario, the desired signal is always present in the sample data, the 100 Monte Carlo simulations are performed, and all the results are shown by averaging. The proposed method is compared with the iterative robust minimum variance beamformer (IRMVB) [8], the robust Capon beamformer (RCB) [4], the eigenspace-based beamformer (ECB) [6], and the reconstruction-based beamformer [12]. From the equation (6), the optimal output SINR can be obtained. The parameter $\varepsilon_{0}=0.1$ and $\eta=10^{-7}$ are used for IRMVB, the optimal $\varepsilon_{1}=\min _{\varphi}\left\|\mathbf{a}_{0} e^{j \varphi}-\overline{\mathbf{a}}_{0}\right\|^{2}$ are set for RCB [4]. The uncertainty level $\varepsilon_{2}=0.5$ is set for the proposed one and $[12]$.

\subsection{Example 1: SV Mismatch due to Gain and Phase Disturbance}

In first example, we consider the influence of gain and phase disturbance on the performance in term of SINR. Considering amplitude disturbance $\alpha_{m}$ and phase disturbance $\varphi_{m}$ of the $m$ th element, which are zero-mean Gaussian variables with standard deviations of 0.1 and $5^{\circ}$, respectively. Therefore, the nominal and the actual SV can be modeled as $\bar{a}(\theta)=\left[1, e^{j \phi_{1}}, \ldots, e^{j \phi_{m-1}}\right]$ and $a(\theta)=\left[1+\alpha_{0},\left(1+\alpha_{1}\right) e^{j\left[\phi_{1}+\varphi_{1}\right]}, \ldots,\left(1+\alpha_{m-1}\right) e^{j\left[\phi_{m-1}+\varphi_{m-1}\right]}\right]$, respectively.

Considering the predefined angle tolerance $\delta$ used by [12], different values are investigated. By setting the number of snapshots $K=50$, the mean output SINRs against the desired signal's SNR is illustrated in Fig.3(a). One can observe that the proposed method has better performance compared with the beamformer in [4][6][8], especially when SNR $\geqslant 10 \mathrm{~dB}$. Although, the superiority of the proposed method over the method [12] is not obvious, from the enlargement of the part corresponding to $\mathrm{SNR}=40 \mathrm{~dB}$, and shown on the top left in Fig.3(a), the propose method is slightly better than it. In addition, 


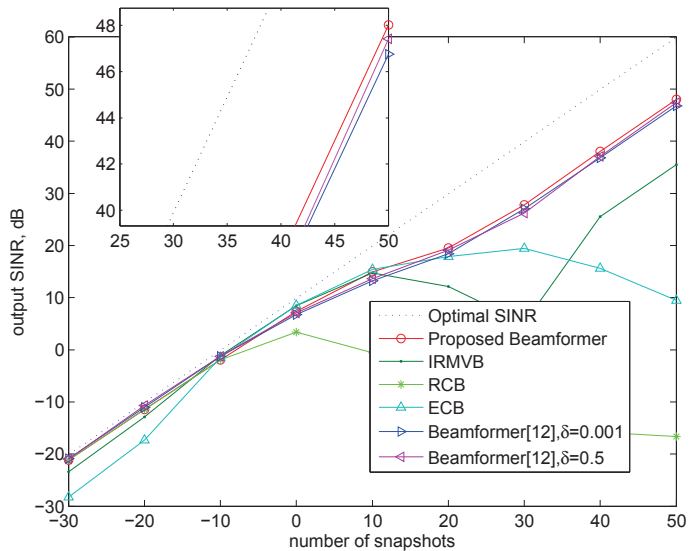

(a)

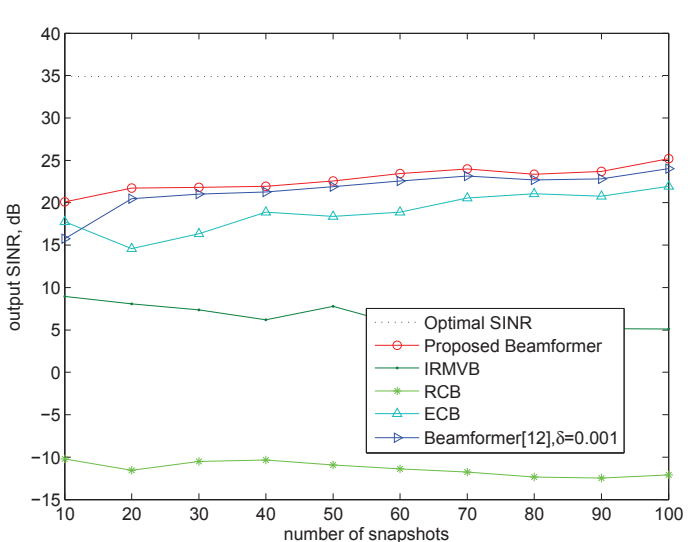

(b)

Figure 3: (a) Output SINRs against SNR (b) Output SINRs against number of snapshots

it can be seen that the performance of the beamformer [12] depends on the predefined angle tolerance $\delta$. In order to show results clearly, we set $\delta=0.001$ in subsequent experiments. From a complexity point of view, in low SNR, the proposed one is only to corrected the desired SVs, but the beamformer [12] pays a high price to reconstruct the INC matrix. As for the high SNR, instead of the whole SVs are corrected in [12], only the main interference SVs are corrected as for the proposed one. Correspondingly, Fig.3(b) demonstrates the mean output SINRs against the number of snapshots, when the input $\mathrm{SNR}$ is fixed at $25 \mathrm{~dB}$. It can be found that the proposed one still outperforms the other beamformers.

\subsection{Example 2: Mismatch due to Incoherent Local Scattering}

In the second example, the incoherent local scattering of the desired signal is considered. Assuming a time-varying spatial nature of desired signal SV, 
it can be modeled as follows

$$
\boldsymbol{x}_{s}(k)=s_{0}(k) \boldsymbol{a}_{0}+\sum_{i=1}^{4} s_{i} \boldsymbol{b}\left(\theta_{i}\right)
$$

where $\boldsymbol{a}_{0}$ denotes the direct path of the desired signal direction $\theta_{0}=2^{\circ}$. The direction $\theta_{i}, i=1,2,3,4$ represents the incoherently scattered signal direction, which is identically distributed and drawn from Gaussian random variables with mean $\theta_{0}$ and standard deviation $2^{\circ}$ in each simulation run. Moreover, the $s_{i}(k)$ are i.i.d. zeros-mean complex Gaussian random variable drawn from $N(0,1)$. It notes that the $\theta_{i}$ changes from run to run, while, it should be frozen from snapshot to snapshot. The expression in (21) corresponds to the case of incoherent local scattering [23]. In this case, the siganl covariance matrix $\boldsymbol{R}_{s}$ is no longer a rank-one matrix, and the equation (6) should be rewritten in a more general form [3]

$$
\text { SINR }=\frac{\boldsymbol{w}^{H} \boldsymbol{R}_{s} \boldsymbol{w}}{\boldsymbol{w}^{H} \boldsymbol{R}_{i+n} \boldsymbol{w}}
$$

Furthermore, the (22) can be maximized by [3]

$$
w_{o p t}=\mathcal{P}\left\{\boldsymbol{R}_{i+n}^{-1} \boldsymbol{R}_{s}\right\}
$$

where $\mathcal{P}\{\cdot\}$ denotes to calculate the principal eigenvector of a matrix.

Fig.4(a) and Fig.4(b) show the performance of the proposed method versus the input SNR and the number of training snapshots, respectively. As can be seen, the proposed method has better performance than IRMVB, ECB, and the beamformer [12] over the wide range of SNR. By contrast, in high SNR, the RCB, IRMVB, ECB suffer a significant performance degradation, 
due to the presence of desired signal component. In practice, in high SNR, the proposed beamformer belongs to the class of INC matrix reconstruction, but the starting point of reconstruction is different from the reconstructionbased beamformer [12].

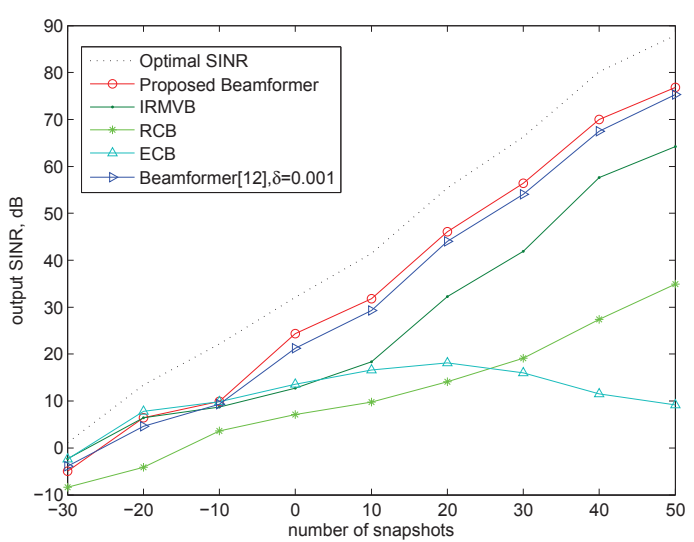

(a)

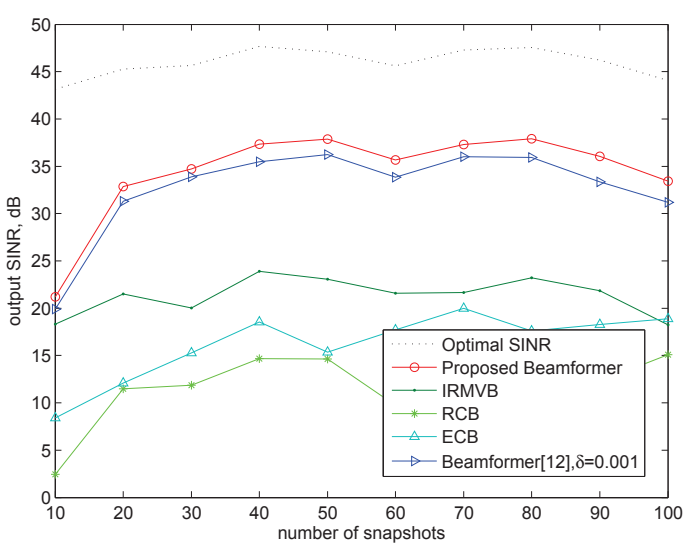

(b)

Figure 4: (a) Output SINRs against SNR (b) Output SINRs against number of snapshots

\subsection{Example 3: Mismatch due to Coherent Local Scattering}

In the third scenario, the impact of the desired signal SV mismatch due to local scattering on output SINR is investigated. Assumed that the desired signal is a plane wave from the $2^{\circ}$, whereas the actual desired signal consists of five signal paths, and can be expressed by

$$
\boldsymbol{a}=\boldsymbol{a}_{0}+\sum_{i=1}^{4} e^{j \psi_{i}} \boldsymbol{b}\left(\theta_{i}\right)
$$


where $\boldsymbol{a}$ and $\boldsymbol{b}\left(\theta_{i}\right)(i=1,2,3,4)$ correspond, respectively, to the direct path and the coherently scattered paths. The $\boldsymbol{b}\left(\theta_{i}\right)$ denotes the SV of the $i$ th path with the direction $\theta_{i}$. For each simulation run, the $\theta_{i}$ can be independently drawn from a uniform random generator with mean $=3^{\circ}$ and standarddeviation $=2^{\circ}$. Correspondingly, in each simulation run, the variables $\psi_{i}(i=1,2,3,4)$ the phases of coherently scattered paths, and can be uniformly obtained from the interval $[0,2 \pi]$. The parameters $\theta_{i}$ and $\psi_{i}$ only change from run to run, while remaining fixed from snapshot to snapshot. The equation (24) is so-called coherent scattering [24].

Similarly, the performance of the proposed method further is shown versus the SNR with the fixed number of snapshots $K=50$ in Fig.5(a), and the the number of snapshots $K$ with the fixed $\mathrm{SNR}=25 \mathrm{~dB}$ in Fig.5(b), respectively. Compared with above examples, the scale of the degradation of performance is different corresponding to various beamformers. However, similar performance have been shown. It can be found that the proposed beamformer still has better performance.

\section{Conclusion}

In this paper, the novel beamformer in view of IF is proposed. The IF is associated with SNR, when the SNR is low, only by estimating the desired $\mathrm{SV}$ without covariance matrix reconstruction, it is robust to SV mismatch. If the SNR is high, besides the desired SV estimation, the INC matrix is also reconstructed based on the Capon spatial spectrum. During the reconstruction, we consider not only the sparsity of interferences direction but also the interference SV mismatches. By using the sparsity, only the interference SVs 


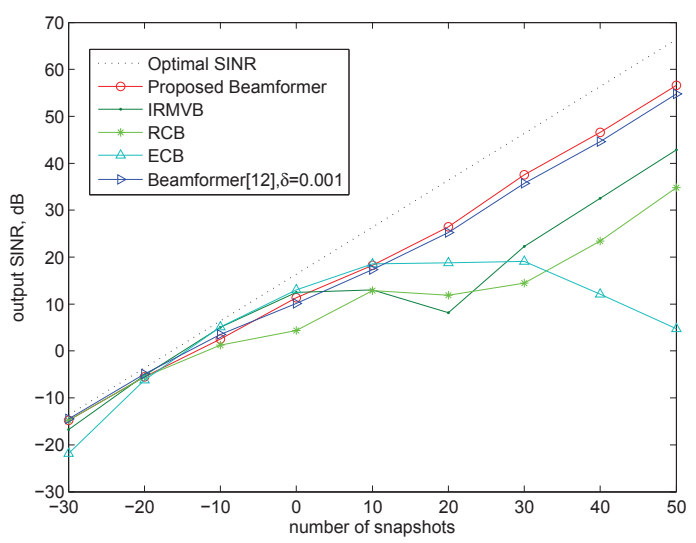

(a)

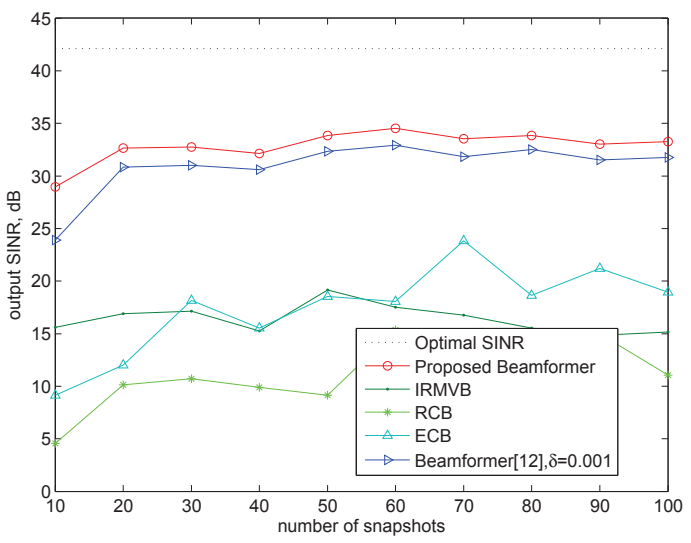

(b)

Figure 5: (a) Output SINRs against SNR (b) Output SINRs against number of snapshots

located in the interference angular sectors are corrected, and then take part in the integration of the spatial spectrum. The simulation results have shown that the proposed beamformer can achieve a better performance in case of coherent local and incoherent local scattering, as well as the SV mismatch resulted from gain and phase disturbance.

\section{Reference}

[1] J. Li, P. Stoica, Robust adaptive beamforming, Wiley Online Library, 2006.

[2] J. Capon, High-resolution frequency-wavenumber spectrum analysis, Proceedings of the IEEE 57 (8) (1969) 1408-1418.

[3] S. A. Vorobyov, A. B. Gershman, Z.-Q. Luo, Robust adaptive beamforming using worst-case performance optimization: A solution to the sig- 
nal mismatch problem, Signal Processing, IEEE Transactions on 51 (2) (2003) 313-324.

[4] J. Li, P. Stoica, Z. Wang, On robust capon beamforming and diagonal loading, Signal Processing, IEEE Transactions on 51 (7) (2003) 1702 1715.

[5] A. Elnashar, S. M. Elnoubi, H. A. El-Mikati, Further study on robust adaptive beamforming with optimum diagonal loading, Antennas and Propagation, IEEE Transactions on 54 (12) (2006) 3647-3658.

[6] D. D. Feldman, L. J. Griffiths, A projection approach for robust adaptive beamforming, Signal Processing, IEEE Transactions on 42 (4) (1994) $867-876$.

[7] A. Khabbazibasmenj, S. A. Vorobyov, A. Hassanien, Robust adaptive beamforming based on steering vector estimation with as little as possible prior information, Signal Processing, IEEE Transactions on 60 (6) (2012) 2974-2987.

[8] S. E. Nai, W. Ser, Z. L. Yu, H. Chen, Iterative robust minimum variance beamforming, Signal Processing, IEEE Transactions on 59 (4) (2011) $1601-1611$.

[9] Y. Gu, A. Leshem, Robust adaptive beamforming based on jointly estimating covariance matrix and steering vector, in: Acoustics, Speech and Signal Processing (ICASSP), 2011 IEEE International Conference on, IEEE, 2011, pp. 2640-2643. 
[10] Y. Gu, N. A. Goodman, S. Hong, Y. Li, Robust adaptive beamforming based on interference covariance matrix sparse reconstruction, Signal Processing 96 (2014) 375-381.

[11] L. Huang, J. Zhang, X. Xu, Z. Ye, Robust adaptive beamforming with a novel interference-plus-noise covariance matrix reconstruction method, Signal Processing, IEEE Transactions on 63 (7) (2014) 1643-1650.

[12] Z. Lu, Y. Li, M. Gao, Y. Zhang, Interference covariance matrix reconstruction via steering vectors estimation for robust adaptive beamforming, Electronics Letters 49 (22) (2013) 1373-1374.

[13] Y. Gu, A. Leshem, Robust adaptive beamforming based on interference covariance matrix reconstruction and steering vector estimation, Signal Processing, IEEE Transactions on 60 (7) (2012) 3881-3885.

[14] W. Jia, W. Jin, S. Zhou, M. Yao, Robust adaptive beamforming based on a new steering vector estimation algorithm, Signal Processing 93 (9) (2013) 2539-2542.

[15] Z. L. Yu, Z. Gu, J. Zhou, Y. Li, W. Ser, M. H. Er, A robust adaptive beamformer based on worst-case semi-definite programming, Signal Processing, IEEE Transactions on 58 (11) (2010) 5914-5919.

[16] L. Landau, R. C. de Lamare, M. Haardt, Robust adaptive beamforming algorithms using the constrained constant modulus criterion, Signal Processing, IET 8 (5) (2014) 447-457. 
[17] Y. Gu, W. Zhu, M. Swamy, Adaptive beamforming with joint robustness against covariance matrix uncertainty and signal steering vector mismatch, Electronics letters 46 (1) (2010) 86-88.

[18] P. Stoica, Z. Wang, J. Li, Robust capon beamforming, IEEE Signal Processing Letters 10 (2003) 172-175.

[19] K. Harmanci, J. Tabrikian, J. L. Krolik, Relationships between adaptive minimum variance beamforming and optimal source localization, Signal Processing, IEEE Transactions on 48 (1) (2000) 1-12.

[20] M. Rahmani, M. H. Bastani, Robust and rapid converging adaptive beamforming via a subspace method for the signal-plus-interferences covariance matrix estimation, Signal Processing, IET 8 (5) (2014) 507520.

[21] H. V. Trees, L. Harry, Optimum array processing: part iv of detection, estimation, and modulation theory, Wiley-Interscience, New York.

[22] A. B. Gershman, M. Rübsamen, M. Pesavento, One-and twodimensional direction-of-arrival estimation: An overview of search-free techniques, Signal Processing 90 (5) (2010) 1338-1349.

[23] O. Besson, P. Stoica, Decoupled estimation of doa and angular spread for a spatially distributed source, Signal Processing, IEEE Transactions on 48 (7) (2000) 1872-1882.

[24] J. Goldberg, H. Messer, Inherent limitations in the localization of a coherently scattered source, Signal Processing, IEEE Transactions on 46 (12) (1998) 3441-3444. 